\title{
Atteintes rénales au cours du déficit en cobalamine $C$
} Kidney disease in cobalamin $\mathrm{C}$ deficiency

\section{Mathilde Lemoine ${ }^{\mathrm{a},{ }^{\star}}$, Steven Grangé ${ }^{\mathrm{b}}$, Dominique Guerrot ${ }^{\mathrm{a}, \mathrm{c}}$}

a Service de néphrologie, dialyse et transplantation, $\mathrm{CHU}$ de Rouen, 1 rue de Germont, 76031 Rouen, France

b Service de réanimation médicale, CHU de Rouen, 1 rue de Germont, 76031 Rouen, France

${ }^{\mathrm{C}}$ Inserm U1096, UFR Médecine Pharmacie, 22 Bd Gambetta, 76183 Rouen, France

\section{* Auteur correspondant} ml.mathildelemoine@gmail.com 


\section{Résumé}

Le déficit en cobalamine $C$ (cblC) est la plus fréquente erreur innée du métabolisme intracellulaire de la vitamine $B_{12}$. Cette maladie autosomique récessive est liée à des mutations du gène $M M A C H C$ codant pour une cyanocobalamine décyanase. Elle entraîne une hyperhomocystéinémie associée à une hypométhioninémie et une acidurie méthylmalonique. Deux formes ont été décrites : les formes précoces, définies par leur survenue avant l'âge d'un an, sont caractérisées par un tableau multisystémique sévère associant retard de croissance et manifestations neurologiques et ophtalmologiques. Elles s'opposent aux formes tardives, moins sévères et très hétérogènes. Les atteintes rénales survenant au cours du déficit en cobalamine $C$ sont une entité rare. Trente-huit cas ont été décrits dans la littérature chez des patients âgés de quelques jours à 28 ans au moment des premiers symptômes. La plupart présentaient un tableau biologique de microangiopathie thrombotique associé à une insuffisance rénale aiguë, avec chez 21 patients la présence d'une microangiopathie thrombotique rénale prouvée sur la biopsie. Le pronostic est sévère, le plus souvent létal en l'absence de traitement et lié à la sévérité de l'atteinte rénale dans les formes précoces. L'évolution est plus favorable dans les formes tardives et un grand nombre de patients est sevré de la dialyse une fois le traitement adapté instauré. Le diagnostic de déficit en cobalamine $C$ doit donc être évoqué devant tout cas de microangiopathie thrombotique rénale, quel que soit l'âge du patient et même en l'absence de symptômes neurologiques, afin de mettre en place rapidement le traitement spécifique.

Mots clés : déficit en cobalamine C; microangiopathie thrombotique; néphropathie congénitale

\section{Abstract}

Cobalamin $\mathrm{C}$ deficiency (cblC) is the most common inborn error of vitamin $\mathrm{B}_{12}$ metabolism. This autosomal recessive disease is due to mutations in MMACHC gene, encoding a cyanocobalamin decyanase. It leads to hyperhomocysteinemia associated with hypomethioninemia and methylmalonic aciduria. Two distinct phenotypes have been described: early-onset forms occur before the age of one year and are characterized by a severe multisystem disease associating failure to thrive to neurological and ophthalmological manifestations. They are opposed to late-onset forms, less severe and heterogeneous. CbIC deficiency-associated kidney lesions remain poorly defined. Thirtyeight cases have been described. Age at initial presentation varied from a few days to 28 years. Most of the patients presented renal thrombotic microangiopathy (TMA) associated with acute renal failure, and 21 patients presented typical lesions of renal thrombotic microangiopathy on kidney biopsy. Prognosis was poor, leading to death in the absence of treatment, and related to the severity of renal lesions in the early-onset forms. Late-onset disease had better prognosis and most of patients were weaned off dialysis after treatment initiation. We suggest that all the patients with renal TMA be screened for cobalamin metabolism disorder, regardless of age and even in the absence of neurological symptoms, to rapidly initiate the appropriate treatment.

Keywords: cobalamin C deficiency; congenital nephropathy; thrombotic microangiopathy

\section{Abréviations}

AdoCbl

BHMT

$\mathrm{Cbl}$ adénosylcobalamine

bétaïne-homocystéine méthyltransférase

cobalamine 


$\begin{array}{lc}\text { CNCbl } & \text { cyanocobalamine } \\ \text { FI } & \text { facteur intrinsèque } \\ \text { HSF } & \text { hyalinose segmentaire et focale } \\ \text { HTAP } & \text { hypertension artérielle pulmonaire } \\ \text { IF } & \text { immunofluorescence } \\ \text { IM } & \text { intramusculaire } \\ \text { IRA } & \text { insuffisance rénale aiguë } \\ \text { IRC } & \text { insuffisance rénale chronique } \\ \text { LDH } & \text { lactate déshydrogénase } \\ \text { MAT } & \text { microangiopathie thrombotique } \\ \text { ME } & \text { microscopie électronique } \\ \text { MeCbl } & \text { méthylcobalamine } \\ \text { MMA } & \text { acidurie méthylmalonique } \\ \text { MMACHC } & \text { methylmalonic aciduria cb/C type with homocytinuria } \\ \text { MO } & \text { microscopie optique } \\ \text { MTRR } & \text { méthionine synthase réductase } \\ \text { NO } & \text { oxyde nitrique } \\ \text { NTIC } & \text { néphropathie tubulo-interstitielle chronique } \\ \text { OHCbl } & \text { hydroxycobalamine } \\ \text { PO } & \text { per os } \\ \text { PTT } & \text { purpura thrombotique thrombocytopénique } \\ \text { ROS } & \text { dérivés réactifs de l'oxygène } \\ \text { SC } & \text { sous-cutané } \\ \text { SHU } & \text { syndrome hémolytique et urémique } \\ \text { TC } & \text { transcobalamine } \\ \text { tPA } & \text { activateur du plasminogène tissulaire }\end{array}$

\section{Introduction}

Le déficit en cobalamine $C$ est la plus fréquente erreur innée du métabolisme de la vitamine $B_{12}$. Les premiers symptômes apparaissent le plus souvent chez le nourrisson ou le jeune enfant, mais il existe également des formes tardives diagnostiquées chez l'adolescent ou le jeune adulte. Peu d'études se sont intéressées aux atteintes rénales survenant au cours du déficit en cobalamine $\mathrm{C}$. II s'agit pourtant d'une maladie rare, mais grave, pour laquelle il existe un traitement efficace. Nous proposons une revue de la littérature des atteintes rénales au cours du déficit en cobalamine $C$ ainsi qu'une mise au point sur cette pathologie bien souvent méconnue des néphrologues.

\section{$\underline{\text { 3. La cobalamine, ou vitamine } B_{12}}$}

\subsection{Physiologie}

La vitamine $B_{12}$, également appelée cobalamine (Cbl), est une vitamine hydrosoluble de 1335 daltons, ce qui en fait la plus grosse et la plus complexe des huit vitamines B. Les cobalamines ont une structure chimique proche de l'hème, dans laquelle l'atome de fer est remplacé par un atome de cobalt (Figure 1). La famille des cobalamines comprend plusieurs formes en fonction du résidu lié à l'ion cobalt, avec chacune des propriétés physiologiques particulières [1] :

- la cyanocobalamine (CNCbl);

- l'hydroxycobalamine (OHCbl) ;

- la méthylcobalamine (MeCbl);

- la 5' déoxy-adénosylcobalamine (AdoCbl). 
La plupart des micro-organismes, comme les algues ou les bactéries, ont la capacité de produire de la vitamine $\mathrm{B}_{12}$ [2], contrairement aux eucaryotes. Les animaux dépendent donc des bactéries et la chaîne alimentaire permet la transmission de cette protéine des herbivores aux carnivores [1]. Chez l'homme, la vitamine $\mathrm{B}_{12}$ provient de la chair des animaux et des produits de leur exploitation (laits, œufs). La Cbl liée aux protéines animales est libérée grâce à l'acidité de l'estomac, puis liée à des glycoprotéines, appelées «protéines $R$ », sécrétées par l'estomac et les glandes salivaires. À l'arrivée dans le duodénum, les protéases pancréatiques digèrent les protéines $R$, ce qui permet la libération de la $\mathrm{Cbl}$ et sa liaison au facteur intrinsèque $(\mathrm{FI})$, une glycoprotéine sécrétée par les cellules pariétales gastriques [3]. Le complexe Cbl-FI est ensuite phagocyté par les entérocytes de l'iléon terminal grâce à la cubiline, un récepteur membranaire spécifique du FI [4]. Au sein de l'entérocyte, la Cbl est liée à la transcobalamine II (TCII) et le complexe Cbl-TCII est libéré dans la circulation portale, puis est endocyté grâce au récepteur de la TCII. Au sein de la cellule, la Cbl est dissociée de la TCll par les protéases du lysosome [5]. La Cbl ainsi libérée dans le cytoplasme contient un atome de cobalt sous forme trivalente (cob(III)alamine) et va être réduite sous forme divalente (cob(II)alamine), puis monovalente (cob(I)alamine) avant d'être transformée en l'une de ses deux formes actives: la méthylcobalamine dans le cytoplasme ou l'adénosylcobalamine dans la mitochondrie (Figure 2). La majorité de la Cbl absorbée est stockée au niveau hépatique, et les réserves sont suffisantes pour plusieurs années.

\subsection{Métabolisme de la cobalamine}

Seules deux formes de la cobalamine jouent un rôle de coenzyme intervenant dans le métabolisme chez l'homme [1] :

- l'adénosylcobalamine (AdoCbl) ;

- la méthylcobalamine (MeCbl).

L'AdoCbl se comporte comme un donneur ou un accepteur d'hydrogène et est notamment cruciale au cours de la transformation du méthylmalonyl-coenzyme $A$ en succinylcoenzyme A médiée par la L-méthylmalonyl-coenzyme A mutase (MMA mutase) dans la mitochondrie.

La MeCbl, quant à elle, joue un rôle dans la méthylation intracytoplasmique de I'homocystéine en méthionine puis en S-adénosyl-méthionine via la méthionine synthase, également appelée 5-méthyltétrahydrofolate-homocystéine méthyltransférase (cycle de méthylation). Elle permet également la conversion du N5-méthyl-tétrahydrofolate en tétrahydrofolate qui joue un rôle crucial dans la synthèse des bases puriques et pyrimidiques, composés essentiels de l'ADN et de l'ARN, ce qui explique le rôle de la vitamine $B_{12}$ au cours de l'hématopoïèse (Figure 3) [1].

Le métabolisme de la vitamine $B_{12}$ peut être perturbé en cas de carence, liée à un défaut d'apport ou d'absorption (maladie de Biermer), ou en cas d'erreur innée du métabolisme intracellulaire de la cobalamine. Ces anomalies génétiques, rares, regroupent l'acidémie méthylmalonique, l'acidémie méthylmalonique avec homocytinurie et l'homocystinurie. Ce sont des pathologies autosomiques récessives liées à des mutations de gènes codant pour des groupes de complémentation (Tableau 1 ; Figure 2) [6].

\section{Le déficit en cobalamine $\mathrm{C}$}

\section{1 Épidémiologie}

L'anomalie du groupe de complémentation $\mathrm{C}$, communément appelée déficit en cobalamine $\mathrm{C}$ ou cblC, est la plus fréquente erreur innée du métabolisme de la cobalamine. Le premier cas a été décrit en 1969 chez un enfant présentant une atteinte neurologique sévère associée à une hyperhomocystéinémie, une hypométhioninémie et 
une acidurie méthylmalonique [7]. Depuis, plus de 500 cas ont été décrits dans la littérature [8].

La prévalence du déficit en cblC est estimée à 1/200 000 naissances [6], mais récemment le dépistage néonatal systématique aux États-Unis a suggéré une prévalence proche de 1/100 000 dans l'État de New-York [9], 1/67 000 en Californie et même 1/46 000 dans la population hispanique [10]. La prévalence est très probablement sous-estimée du fait d'une grande hétérogénéité clinique.

\subsection{Physiopathologie}

Le déficit en cblC est lié à une mutation du gène MMACHC (Methylmalonic aciduria cb/C type with homocytinuria) qui code pour la synthèse d'une cyanocobalamine décyanase. Celle-ci joue un rôle de chaperonne intracellulaire pour la $\mathrm{Cbl}$ et catalyse la réaction de décyanation de la $\mathrm{CNCbl} \mathrm{[11].} \mathrm{Le} \mathrm{déficit} \mathrm{en} \mathrm{cblC} \mathrm{entraîne} \mathrm{alors} \mathrm{une} \mathrm{diminution} \mathrm{de} \mathrm{la}$ conversion de la cob(III)alamine en en cob(II)alamine et donc une diminution de synthèse de ses deux formes métaboliquement actives, la MeCbl et l'AdoCbl (Figure 2). Le déficit en ces deux coenzymes est la cause de l'accumulation d'homocystéine et d'acide méthylmalonique ainsi que de la diminution de la synthèse de méthionine (Figure 4), trois anomalies qui vont entraîner une symptomatologie liée à des mécanismes synergiques [12].

II a été récemment montré que la protéine codée par le gène $M M A C H C$ avait également une activité alkyltransférase catalysant la réaction de déalkylation des alkylcobalamines présentes dans l'alimentation, permettant ainsi leur conversion en MeCbl et AdoCbl $[13,14]$.

\subsection{Diagnostic clinique}

\subsubsection{Présentation clinique}

La présentation clinique du déficit en cblC varie en fonction de l'âge d'apparition des symptômes et est très hétérogène, ce qui peut expliquer le fréquent retard diagnostique, notamment dans les formes dites tardives.

Les formes les plus sévères, prénatales, peuvent être révélées par un retard de croissance intra-utérin, une dysmorphie, des malformations cardiaques ou une microcéphalie [15].

Les formes précoces, les plus fréquentes, représentent près de $90 \%$ des cas rapportés $[8,16]$. Elles surviennent typiquement avant l'âge d'un an et sont caractérisées par une atteinte multi-systémique associant retard de croissance, atteinte neurologique et oculaire, mais aussi anomalies hématologiques, gastro-intestinales et cardio-pulmonaires $[8,16]$.

Les formes tardives, définies par leur survenue après l'âge d'un an, sont plus rares et généralement moins graves. Elles sont hétérogènes et peuvent être révélées chez les jeunes enfants par une hypertension artérielle pulmonaire (HTAP) ou un syndrome hémolytique et urémique (SHU), et chez les enfants plus âgés et les adolescents par des troubles neuropsychiatriques, une ataxie ou une myélopathie. Les événements thromboemboliques surviennent le plus souvent dans les formes découvertes à l'âge adulte $[8,12,15,17]$.

L'hétérogénéité clinique semble en grande partie dépendre du type de mutation du gène MMACHC, mais d'autres facteurs sont probablement impliqués, tels que le régime alimentaire, l'environnement, ou encore la présence de variations génétiques d'autres gènes [15].

\subsubsection{Atteintes d'organes}




\subsubsection{Système nerveux}

Les atteintes du système nerveux sont quasiment constantes au cours des déficits en cblC. Elles se traduisent dans les formes précoces par des crises convulsives, une hydrocéphalie ou des troubles cognitifs.

En cas d'épilepsie, l'électroencéphalogramme retrouve un tracé de crises partielles ou complètes non spécifique [18]. L'analyse en résonance magnétique, lorsqu'elle est réalisée, peut révéler une hydrocéphalie, des anomalies de la substance blanche à types d'hypersignaux T2 péri-ventriculaires et/ou une atrophie cérébrale [17,19]. L'examen anatomopathologique post-mortem retrouve une démyélinisation diffuse et un infiltrat macrophagique [20].

Au cours des formes tardives, peut également survenir un tableau de sclérose subaiguë combinée de la moelle [21], similaire à l'atteinte caractéristique du déficit en vitamine $B_{12}$.

\subsubsection{Manifestations ophtalmologiques}

Les anomalies oculaires survenant au cours du déficit en cblC sont d'autant plus sévères et fréquentes que l'âge d'apparition des symptômes est précoce [22]. La maculopathie peut être révélée par un nystagmus de survenue précoce et le fond d'œil retrouve un aspect typique de maculopathie « en œil de bœuf » [20]. Certains patients développent une rétinopathie à un degré variable, allant de la perte de la couche de fibres nerveuses de la rétine à l'atrophie maculaire et optique avancée [23]. L'électrorétinogramme, qui permet d'objectiver la dégénérescence rétinienne [24], est recommandé dans le suivi des patients atteints d'un déficit en cblC [25].

\subsubsection{Manifestations hématologiques}

Les cytopénies telles que l'anémie mégaloblastique, la thrombopénie ou la neutropénie, sont liées au déficit en $\mathrm{MeCbl}$, coenzyme nécessaire à la production de tétrahydrofolate et donc à la synthèse de l'ADN [20]. Elles sont fréquemment associées aux autres manifestations et peuvent orienter le diagnostic.

\subsubsection{Manifestations vasculaires}

L'hyperhomocystéinémie entraîne un risque thrombotique accru lié à des altérations de la fonction endothéliale, se traduisant par la survenue de thromboses veineuses profondes récurrentes [26,27], d'embolies pulmonaires [26,28], d'accidents vasculaires cérébraux $[29,30]$ ou d'une hypertension artérielle pulmonaire (HTAP) [31]. L'HTAP, de type précapillaire, peut être au premier plan clinique [32,33]. Les données autopsiques rapportent la présence de lésions de maladie veino-occlusive [34].

\subsubsection{Autres manifestations}

Les autres manifestions décrites chez les patients souffrant d'un déficit en cblC sont les manifestations cardiaques (insuffisance cardiaque congénitale, cardiomyopathie dilatée, malformations [35]), la stéatose hépatique, la gastrite, les entéropathies ou encore la desquamation cutanée [20]. Enfin, le déficit en cblC peut s'accompagner d'atteintes rénales, le plus souvent microangiopathiques, qui font l'objet d'un chapitre spécifique.

L'ensemble des manifestations survenant au cours des déficits en cobalamine $\mathrm{C}$ sont résumées dans le tableau 2.

\subsection{Diagnostic biologique}

\subsubsection{Paramètres biochimiques}

La suspicion diagnostique de déficit en cblC se fait sur la présence d'une hyperhomocystéinémie, généralement supérieure à $100 \mu \mathrm{mol} / \mathrm{L} \quad(\mathrm{N}=3-13 \mu \mathrm{mol} / \mathrm{L})$, 
associée à une acidémie méthylmalonique (>100 $\mu \mathrm{mol} / \mathrm{L}$ pour $\mathrm{N}<0,27 \mu \mathrm{mol} / \mathrm{L}$ ), une acidurie méthylmalonique (> 100 à $1000 \mathrm{mmol} / \mathrm{mol}$ de créatinine pour $\mathrm{N}<4 \mathrm{mmol} / \mathrm{mol}$ ) et une méthioninémie effondrée ( $\mathrm{N}=11-37 \mu \mathrm{mol} / \mathrm{L})$ [6].

Dans les formes tardives, la méthioninémie peut être plus élevée et l'homocystéinémie plus basse, ce qui suggère une corrélation entre sévérité clinique et paramètres biochimiques [8].

Il est important de noter que le taux plasmatique de vitamine $B_{12}$ est normal et permet d'éliminer le diagnostic différentiel de carence en vitamine $\mathrm{B}_{12}$ par défaut d'apport ou d'absorption [15].

Les dosages doivent être renouvelés après l'introduction du traitement afin de confirmer l'efficacité thérapeutique, mais également à distance lors de tout épisode aigu ou de modification clinique.

\subsection{2 Études de complémentation}

Lorsque le diagnostic d'erreur innée du métabolisme de la cobalamine est suspecté sur les paramètres biochimiques, la réalisation d'études de complémentation sur culture de fibroblastes permet de distinguer le groupe de complémentation en cause.

Les fibroblastes du patient sont cultivés avec des fibroblastes issus de différentes lignées cellulaires, définies par leur déficience en un groupe de complémentation. L'incorporation du propionate marqué est alors étudiée dans chaque culture, et le groupe de complémentation en présence duquel il n'y a pas d'augmentation de l'incorporation de propionate correspond au groupe de complémentation déficient chez le patient [15].

Peu utilisées depuis l'identification du gène $M M A C H C$, les études de complémentation gardent tout leur intérêt lorsque l'analyse génétique ne permet pas de mettre en évidence de mutation malgré une présomption clinique et biochimique forte.

\subsection{3 Étude génétique}

Le diagnostic de certitude devant une suspicion de déficit en cblC est génétique.

Le gène $M M A C H C$, situé sur le bras court du chromosome 1, a été identifié en 2006 [36]. Son séquençage est maintenant disponible en routine et est recommandé en première intention car plus rapide et moins coûteux que les tests de complémentation [15].

À l'heure actuelle, 87 mutations différentes ont été recensées [37]. La mutation la plus fréquente, représentant $40 \%$ des allèles mutés, est la duplication c.271dupA [36,38], qui entraîne un décalage du cadre de lecture et la formation d'un codon STOP prématuré [39]. Les mutations c.394C>T et c.331C>T sont également fréquemment observées [38].

Certaines études suggèrent une corrélation entre le phénotype et le génotype, qui serait liée à la stabilité de l'ARN messager ou encore à la fonction résiduelle de la protéine. Ainsi, les mutations c.271dupA et c.331C>T apparaissent liées aux formes sévères et précoces $[38,39]$ tandis que la mutation c.394C>T est plus souvent rencontrée dans les formes tardives [36,38,39].

\subsection{Prise en charge}

Le traitement du déficit en cblC est une urgence et il doit être instauré dès la suspicion clinique, après réalisation des dosages biochimiques et sans attendre le résultat de l'analyse génétique. L'objectif est d'améliorer la symptomatologie clinique, de normaliser le taux de méthionine et de diminuer les taux d'homocystéinémie et d'acidémie méthylmalonique autant que possible.

\subsubsection{Hydroxycobalamine}

Le traitement recommandé en cas de déficit en cbIC est l'administration parentérale quotidienne d'hydroxycobalamine $(\mathrm{OHCbl})$, afin de pallier le déficit d'activité enzymatique 
de la cobalamine décyanase [15]. La protéine MMACHC mutante aurait une affinité élevée pour l'OHCbl, en opposition à une perte d'affinité pour la cyanocobalamine (CNCbl) [40], et serait stabilisée en présence de fortes concentrations de cobalamine intracellulaire [41]. La forme intramusculaire (IM) est plus efficace que la forme sous-cutanée (SC), mais est parfois contre-indiquée en cas de traitement anticoagulant, fréquent dans cette population à haut risque thrombo-embolique. À l'heure actuelle, il n'existe aucun consensus concernant la dose à administrer, mais des études suggèrent l'intérêt de doses élevées pour un meilleur contrôle clinico-biologique $[28,42]$, avec un objectif de vitamine $B_{12}$ plasmatique proche de 1000000 pg/mL. Une revue de 2012 suggère également l'intérêt de monter les doses d'OHCbl jusqu'à passer sous un seuil d'homocystéinémie de $45 \mu \mathrm{mol} / \mathrm{L}$ afin de limiter le risque de complications vasculaires [20].

La CNCbl n'a pas d'indication dans cette pathologie puisque le déficit enzymatique ne permet pas la décyanation de cette forme, nécessaire à l'élaboration des deux coenzymes métaboliquement actives. De même, les formes orales (PO) ne sont pas indiquées car inefficaces $[12,20]$.

\subsubsection{Autres thérapeutiques}

La bétaïne, ou N,N,N-triméthylglycine, est également recommandée dans le traitement du déficit en cblC puisqu'elle contribue à la diminution de l'homocystéinémie et à l'augmentation de la méthioninémie. En effet, en tant que donneur de groupe méthyl, son administration permet la reméthylation de l'homocystéine en méthionine via la bétaïnehomocystéine méthyltransférase (BHMT) par une voie indépendante de la méthionine synthase $[12,15]$.

Enfin, l'acide folinique permet également la diminution de l'homocystéinémie et l'augmentation de la méthioninémie en stimulant la voie de la reméthylation [12].

Le régime pauvre en protide n'est pas recommandé au cours des déficits en cblC.

Enfin, l'utilisation du NO au cours des anesthésies est contre-indiquée compte tenu de son effet inhibiteur de la méthionine synthase et de son rôle dans l'épuisement des stocks de vitamine B12 [43].

\section{6 Évolution}

En l'absence de traitement, l'évolution du déficit en cblC est le plus souvent fatale. La mortalité globale est difficile à estimer en raison de la rareté de cette pathologie. Dans une revue de 1997 sur 50 patients, Rosenblatt et al. rapportaient une mortalité de $30 \%$ [16], tandis qu'en 2013, Fisher et al. rapportaient 11,4\% de décès sur une série de 88 patients [8]. L'amélioration de la survie entre ces deux revues peut être expliquée par une meilleure connaissance de cette pathologie, un diagnostic plus précoce et une meilleure prise en charge.

En effet, le traitement rapidement instauré et correctement effectué permet la diminution de l'homocystéinémie, la disparition de l'acidurie méthylmalonique et l'augmentation de la méthioninémie. La correction des anomalies biochimiques est cependant fréquemment incomplète, notamment dans les formes sévères [17]. L'amélioration clinique sous traitement reste aussi bien souvent incomplète [12] puisque seuls les symptômes extraneurologiques disparaissent après la mise en route du traitement [8]. Le pronostic dépend également de la rapidité d'instauration du traitement $[17,20]$.

Le pronostic des formes précoces est extrêmement délétère puisque la majorité des patients décède $[8,16]$, et les quelques survivants présentent une évolution des symptômes neurologiques et ophtalmologiques malgré le traitement [8], même en cas de prise en charge précoce [9]. Bien que moins connu, le pronostic des formes tardives semble être plus favorable $[8,16]$. 


\section{Microangiopathies thrombotiques au cours du déficit en cobalamine C}

\subsection{Généralités sur les microangiopathies thrombotiques}

Le syndrome de microangiopathie thrombotique (MAT) est défini par l'association d'une anémie hémolytique mécanique, d'une thrombopénie de consommation et d'une atteinte d'organe liée à des occlusions microvasculaires systémiques, touchant particulièrement le rein, le cerveau et le myocarde [44]. Au cours de cette pathologie, les lésions endothéliales et les thrombi formés au sein des petits vaisseaux provoquent de fortes contraintes de cisaillement menant à une agrégation plaquettaire et à la destruction mécanique des globules rouges circulants.

Les deux formes typiques de MAT sont le purpura thrombotique thrombocytopénique (PTT) et le syndrome hémolytique et urémique (SHU) dont les étiologies sont multiples : SHU typique, SHU atypique, MAT secondaires [45]. Le PTT est lié à un déficit, le plus souvent acquis, en ADAMTS 13, une métalloprotéase chargée de cliver les multimères de facteur de Willebrand. La symptomatologie prédomine au niveau neurologique du fait d'une atteinte préférentielle des cellules endothéliales cérébrales. Le SHU typique est dû à la production de shigatoxines par un bacille Gram négatif, le plus souvent Escherichia coli O157:H7, et est la première cause d'insuffisance rénale aiguë chez l'enfant [46]. Au cours du SHU typique, l'atteinte rénale est au premier plan et succède à la survenue d'un tableau digestif associant diarrhées fébriles, rectorragies et douleurs abdominales. Les SHU atypiques sont liés à des anomalies innées ou acquises de la voie alterne du complément [47]. D'autres causes de MAT existent, telles que l'hypertension artérielle maligne, les connectivites, ou encore les MAT toxiques, paranéoplasiques ou survenant au cours de la grossesse $[45,47,48]$. Les causes de MAT héréditaires regroupent le PTT congénital, une grande partie des SHU atypiques et certaines anomalies du métabolisme de la cobalamine (Tableau 3).

\subsection{Revue de la littérature}

Plusieurs cas de SHU avec insuffisance rénale aiguë (IRA) ont été décrits chez des patients atteints de déficit en cblC, et des études récentes ont estimé que 10 à $20 \%$ des patients atteints d'un déficit en cblC développaient un SHU, et ce, plus fréquemment dans les formes tardives [8,17].

La première description d'un cas de MAT dans un contexte de déficit en cblC remonte à 1979 [49]. II s'agissait d'un enfant âgé de 3 mois présentant un tableau clinico-biologique de MAT associé à une hyperhomocystéinémie avec acidurie méthylmalonique. L'enfant décédait malgré l'introduction d'un traitement par CNCbI IM. L'autopsie retrouvait des lésions rénales typiques de MAT artériolo-glomérulaire associant thrombi glomérulaires et artériolaires, endartérite fibroproliférative en bulbe d'oignon et doubles contours de la membrane basale glomérulaire. L'étude en ME révélait la présence de dépôts sousendothéliaux granuleux.

Les tableaux 4 et 5 rapportent l'ensemble des cas d'atteintes rénales dans un contexte de déficit en cblC retrouvés dans la littérature, ainsi que deux cas non publiés.

\subsection{Présentation clinique et biologique}

Nous avons identifié 38 patients présentant une atteinte rénale dans un contexte de déficit en cblC, dont la plupart (36/38) présentaient un tableau de MAT. L'âge des patients au diagnostic varie de 15 jours à 28 ans, et 15 présentent une atteinte précoce.

Parmi les 30 patients pour lesquels le taux de plaquettes est disponible, seuls 19 ont une thrombopénie au diagnostic, tandis que 36 patients présentent des stigmates d'hémolyse. La plupart des patients ont une dégradation de la fonction rénale et 11 nécessitent le recours à l'épuration extra-rénale. 
La présentation clinique est variable et associe fréquemment une hémolyse et une insuffisance rénale aiguë. Elle est parfois atypique puisqu'en 2015 a été décrit le cas d'un enfant se présentant avec une hypertension artérielle associée à un syndrome néphrotique sans stigmates d'hémolyse [50]. Enfin, il est important de noter que les troubles neurologiques, fréquents dans cette pathologie, ne sont pas toujours présents, et leur absence ne doit surtout pas faire exclure le diagnostic [27,51].

La protéinurie est variable, parfois absente, pouvant aller jusqu'au syndrome néphrotique.

L'HTAP doit également être recherchée lors du diagnostic puisqu'elle apparaît fréquemment associée aux MAT dans notre étude (8 patients avec une présentation tardive).

\subsubsection{Corrélation phénotype-génotype}

Pour 25 patients, l'analyse génétique a permis de confirmer le diagnostic. La mutation c.271dupA est la plus fréquemment retrouvée, à l'état homozygote chez 4 patients avec une présentation précoce, et à l'état hétérozygote chez 13 patients, dont 11 avec une présentation tardive. Les autres mutations communément retrouvées sont :

- la substitution c.276G>T (ou G>A) (5 cas) ;

- la mutation c.565C>A (3 cas dont 2 à l'état homozygote) ;

- la mutation de site d'épissage c.82-9delTTTC (5 cas).

II est intéressant de noter que la mutation c.394C>T, habituellement fréquente dans les formes tardives, n'est retrouvée chez aucun patient présentant une atteinte rénale.

Dans une revue de 2006 recensant 37 patients atteints d'un déficit en cblC, la mutation c.82-9delTTTC était retrouvée chez les 3 patients avec une présentation tardive et un SHU [39]. Les auteurs suggèrent que cette mutation pourrait abolir un site d'épissage spécifique nécessaire à la génération d'une isoforme de la protéine MMACHC essentielle au maintien de l'intégrité rénale.

\subsubsection{Prise en charge et évolution}

Le pronostic du SHU associé au déficit en cblC de forme précoce est très mauvais, avec un fort taux de mortalité au cours de la première année de vie [20,52]. Parmi les 15 cas recensés dans la littérature, 7 sont décédés (47\%):

- deux n'ont pas été traités [53] ;

- deux ont été traités par CNCbl [31,49] ;

- un a été traité tardivement par OHCbl parentérale [53] ;

- un a été traité mais le traitement n'a pas été détaillé [30].

Enfin, un patient a initialement répondu à l'OHCbl parentérale mais est décédé de complications neurologiques [54]. Parmi les 8 survivants, la MAT a disparu sous OHCbl parentérale et aucune récidive n'a été décrite.

Le pronostic des SHU associés aux formes tardives semble plus favorable puisque chez la plupart des patients le traitement par OHCbl par voie IM permet la correction des anomalies hématologiques et la normalisation de la fonction rénale. Parmi les 23 patients recensés, 6 sont décédés ( $26 \%$ ), dont 3 n'avaient reçu aucun traitement, 1 avait été traité par cobalamine orale et 2 par OHCbl IM. La rapidité de la prise en charge apparaît cruciale dans l'évolution [51].

Le pronostic des formes précoces semble être lié à la sévérité de l'atteinte rénale puisque parmi les trois patients dialysés, deux sont décédés. Au contraire, dans les formes tardives, parmi les 10 patients ayant eu recours à l'épuration extra-rénale, un seul est décédé $(10 \%)$, trois ont récupéré une fonction rénale satisfaisante après introduction du traitement adapté et quatre n'ont jamais récupéré, mais trois d'entre eux ont été transplantés. Aucun cas de rechute n'a été décrit. 
Au cours du suivi, la surveillance de l'haptoglobine et des LDH permet de rechercher des signes de MAT chronique [55], qui, s'ils sont présents, doivent faire discuter l'observance du traitement ou l'augmentation de la posologie d'OHCbl [28], mais aussi une autre cause de MAT associée [27].

\subsection{Physiopathologie}

Les mécanismes physiopathologiques impliqués dans les MAT survenant au cours du déficit en cblC ne sont pas encore complètement établis, mais les lésions endothéliales semblent jouer un rôle critique.

Plusieurs études s'accordent sur le rôle probable de l'hyperhomocystéinémie dans la toxicité endothéliale glomérulaire et vasculaire. En effet, elle entraînerait une dysfonction et une activation endothéliale via l'augmentation des ROS [56], mais aussi une activation de la coagulation et de l'agrégation plaquettaire via divers mécanismes. Des études ont montré que l'homocystéine altérait les propriétés anti-thrombotiques de l'endothélium via la diminution de l'inhibition de l'agrégation plaquettaire médiée par le NO [57,58], de l'activation de la protéine $C$ [59] dépendante de la thrombomoduline [60], et de la liaison du tPA à l'endothélium [61,62]. Elle favoriserait également l'activation de molécules procoagulantes exprimées par les cellules endothéliales, telles que le facteur $V$ [63] et le facteur tissulaire [64]. L'homocystéine à des concentrations supra-physiologiques a aussi une toxicité endothéliale $[65,66]$ et engendrerait un détachement des cellules endothéliales in vitro et in vivo [67].

Enfin, l'hyperhomocystéinémie entraînerait également une activation des voies proinflammatoires et l'accumulation intracellulaire de radicaux libres [68]. De plus, l'homocystéine thiolactone, métabolite de l'homocystéine, favoriserait aussi l'accumulation intracellulaire de radicaux libres en interférant avec les mécanismes de phosphorylation oxydative [69], et activerait l'agrégation plaquettaire [70]. L'hypothèse de la toxicité endothéliale de l'hyperhomocystéinémie est appuyée par les lésions vasculaires découvertes lors d'autopsies, telles que les lésions dégénératives des cellules endothéliales pulmonaires [53], la prolifération intimale ou les micro-infarctus [30].

Cependant, d'autres facteurs semblent nécessaires au développement d'une MAT au cours des déficits en cblC. En effet, aucun cas de SHU n'a été rapporté au cours des déficits en cystathionine synthétase, qui associent une hyperhomocystéinémie à une hyperméthioninémie et au cours desquelles surviennent fréquemment des événements thrombo-emboliques veineux [71]. De même, d'autres anomalies du métabolisme intracellulaire de la cobalamine telles que le déficit en cobalamine D ou en TCII ne s'accompagnent pas de stigmates de MAT. L'hypométhioninémie jouerait donc probablement un rôle dans l'atteinte préférentielle des petits vaisseaux.

Une autre hypothèse est celle d'un facteur toxique circulant. En effet, l'efficacité des échanges plasmatiques dans certains cas suggère la clairance d'un métabolite toxique. Ce facteur pourrait être dû à la rétention d'un métabolite habituellement transformé par la cyanocobalamine décyanase qui jouerait lui aussi un rôle dans la toxicité endothéliale [27]. Le lien entre l'acidémie méthylmalonique et la survenue d'une MAT est plus controversé car des cas de SHU ont été décrits au cours des déficits en cobalamine G [72], pathologie caractérisée par une hyperhomocystéinémie avec hypométhioninémie, mais sans acidurie méthylmalonique.

L'hypothèse de l'implication directe du déficit en cblC dans les lésions endothéliales vasculaires via un mécanisme intracellulaire n'a jamais été étudiée.

Chez certains patients sont également retrouvés des facteurs favorisants, notamment une anomalie associée de la voie alterne du complément, telle qu'une mutation du CD46 $[55,73]$ ou du facteur $\mathrm{H}[27]$. 
Enfin, des études suggèrent que la MAT diffuse, à l'origine du SHU, serait probablement aussi à l'origine de l'hydrocéphalie [74].

\section{5 Étude histopathologique comparative}

Afin de mieux caractériser les lésions rénales au cours du déficit en cobalamine $C$, nous avons réalisé une étude histologique comparative entre 7 patients atteints d'un déficit en cobalamine $\mathrm{C}$ et 16 patients d'âge comparable souffrant d'une microangiopathie thrombotique d'une autre étiologie. Chaque biopsie a été relue de manière centralisée et nous avons constaté que les patients présentant une atteinte rénale dans un contexte de déficit en cobalamine $C$ présentaient tous des lésions typiques de MAT glomérulaire et artériolaire. De plus, ces patients présentaient un aspect vacuolisé de la membrane basale glomérulaire ainsi que des dépôts glomérulaires pariétaux d'IgM. L'étude en microscopie électronique confirmait l'aspect vacuolisé de la MBG dû à des dépôts granuleux endomembraneux et des expansions mésangiales. Après comparaison avec la population contrôle, l'aspect vacuolisé de la MBG et les dépôts d'IgM apparaissaient significativement plus fréquents chez les patients atteints d'un déficit en cobalamine C [75].

\section{Atteintes glomérulaires atypiques au cours du déficit en cobalamine $\mathbf{C}$}

Comme l'illustre le tableau 4, les lésions de MAT sont les lésions le plus souvent décrites, mais il existe également quelques cas d'atteinte glomérulaire atypique dans un contexte de déficit en cblC.

La plus ancienne description date de 1990 [31]. Les auteurs rapportaient un cas d'HTAP fatale chez un enfant âgé de 4 mois atteint d'un déficit en cblC. L'examen histologique rénal retrouvait des lésions de hyalinose segmentaire et focale (HSF). Plus récemment, Brunelli et al. ont rapporté le cas d'un adolescent de 16 ans chez qui le diagnostic de déficit en cblC était fait dans un contexte de troubles cognitifs associés à une hématurie [29]. La biopsie rénale retrouvait un aspect de glomérulonéphrite membrano-proliférative (GNMP) et des dépôts glomérulaires d'lgM et C3. Dans les deux cas, il s'agissait de lésions glomérulaires non spécifiques, qui pourraient survenir secondairement dans un contexte de MAT chronique.

\section{Atteintes rénales au cours des autres anomalies du métabolisme de la cobalamine}

\subsection{MAT et autres anomalies du métabolisme de la cobalamine}

En 1999, Labrune et al. ont publié un cas de MAT glomérulaire et artériolaire chez un nourrisson âgé de 18 mois atteint de déficit en cobalamine $E$ (cblE) [72]. La biopsie rénale réalisée dans un contexte d'IRA et d'anémie hémolytique retrouvait des thrombi glomérulaires et artériolaires. Sous traitement par $\mathrm{OHCbl}$ parentérale, la fonction rénale initialement stable se dégradait ensuite progressivement jusqu'à l'insuffisance rénale terminale. L'enfant présentait également une HTAP. Le déficit en cblE est caractérisé par un déficit en méthionine synthase réductase (MTRR) et associe une hyperhomocystéinémie à une hypométhioninémie, sans acidurie méthylmalonique. Plus récemment, un cas de glomérulopathie atypique révélant un déficit en cbIE a également été décrit [76]. L'analyse en microscopie optique retrouvait une prolifération mésangiale avec un aspect lobulé des glomérules, un épaississement des boucles capillaires et des dédoublements membranaires, tandis que la microscopie électronique objectivait la présence de dépôts sous-endothéliaux. À aucun moment n'étaient retrouvés de stigmates biologiques de microangiopathie.

En 2017, a été également décrit un cas de MAT dans un contexte de déficit en méthionine synthase (groupe de complémentation $\mathrm{G}$ ) chez un enfant de 21 mois [77]. 
Enfin, des cas de pseudo-MAT au cours des carences en vitamine B12 ont été décrits avec une prévalence estimée de 2,5\% [78-80].

Aucun cas de MAT au cours des aciduries méthylmaloniques isolées n’a été rapporté.

\subsection{Acidurie méthylmalonique et tubulopathies}

Quatre causes d'acidurie méthylmalonique (MMA) isolée ont été décrites : deux résultant d'un défaut d'activité de la méthylmalonyl-coenzyme A mutase complet (mut0) ou non (mut-) et deux d'un défaut de synthèse de son cofacteur, l'AdoCbl (cblA et cblB).

Des acidoses tubulaires rénales, des néphrites interstitielles et des insuffisances rénales chroniques dues à l'acidurie méthylmalonique ont été décrites au cours des déficits en cbl $A$ et $B$ et de l'acidurie méthylmalonique résistante à la vitamine $B_{12}$ de type mut0 $[81,82]$, et plus rarement mut- [83].

Le premier cas rapporté date de 1989. II s'agissait d'un enfant de 4 ans présentant une IRA et dont la biopsie révélait une néphropathie tubulo-interstitielle chronique (NTIC). Le tableau clinique et biologique s'améliorait après contrôle des anomalies métaboliques [84]. Cette étude rapportait également 12 cas parmi lesquels 8 souffraient d'insuffisance rénale, dont 5 avec un DFG inférieur à $40 \mathrm{~mL} / \mathrm{min} / 1,73 \mathrm{~m}^{2}$ et 1 présentait une diminution du taux de réabsorption du phosphate.

Deux ans plus tard, D'Angio et al. ont rapporté le cas de 7 enfants atteints de MMA isolée dont 6 présentaient une diminution du DFG, 5 un défaut de concentration des urines et 3 un taux de réabsorption du phosphate bas [85].

Plus récemment, dans une série française de 30 patients atteints de MMA isolée, près de la moitié développait une insuffisance rénale chronique (IRC) [86]. Les patients atteints de l'anomalie mut0 présentaient le tableau rénal le plus précoce et le plus sévère. Une autre étude sur 83 patients a montré que I'IRC survenait plus fréquemment chez les patients mut0 $(61 \%)$ ou cblB (66 \%) que chez les patients cblA (21\%) ou mut- $(0)$ [87].

Dans ces différentes études, le degré d'atteinte rénale semble lié à la sévérité de la maladie, et notamment à l'excrétion d'acide méthylmalonique [82,84,87].

La cause de I'IRC chez ces patients est une néphrite tubulo-interstitielle dont la physiopathologie reste encore incomprise [85,88]. Les patients présentent des anomalies tubulaires distales et proximales, telles qu'une diminution de la réabsorption du phosphate, une diminution des capacités de concentration des urines ou une acidose tubulaire proximale [89] ou distale [81]. L'une des hypothèses physiopathologiques est la toxicité de l'acide méthylmalonique excrété sur les cellules tubulaires, mais les mécanismes précis restent à définir. Le rôle d'une dysfonction mitochondriale a également été évoqué dans une étude in vitro sur des cellules tubulaires proximales [90].

Enfin, une protéinurie tubulaire typique apparaît chez les patients atteints du syndrome d'Imerslund-Gräsbeck, un déficit en cubiline entraînant une malabsorption de cobalamine [91].

\section{Conclusion}

Le diagnostic de déficit en cobalamine $\mathrm{C}$ doit être évoqué devant tout cas de microangiopathie thrombotique rénale, quel que soit l'âge du patient et même en l'absence de symptômes neurologiques. En effet, le dosage de l'homocystéine total est simple et peu coûteux, et il permet d'orienter rapidement le diagnostic afin de mettre en route sans tarder le traitement adapté.

\section{Références}

[1] Food and Agriculture Organization, World Health Organization. 2001. Human vitamin and mineral requirements. www.fao.org/3/a-y2809e.pdf 
[2] Roth JR, Lawrence JG, Bobik TA. Cobalamin (coenzyme B12): synthesis and biological significance. Annu Rev Microbiol. 1996;50:137-81.

[3] Festen HP. Intrinsic factor secretion and cobalamin absorption. Physiology and pathophysiology in the gastrointestinal tract. Scand J Gastroenterol Suppl. 1991;188:1-7.

[4] Alpers DH. Absorption and blood/cellular transport of folate and cobalamin: Pharmacokinetic and physiological considerations. Biochimie. 2016;126:52-6.

[5] Cooper BA, Rosenblatt DS. Inherited defects of vitamin B metabolism. Annu Rev Nutr. 1987;7:291-320.

[6] Carrillo N, Adams D, Venditti CP. Disorders of intracellular cobalamin metabolism. www.ncbi.nlm.nih.gov/books/NBK1328/

[7] Mudd SH, Levy HL, Abeles RH, Jennedy JP. A derangement in B12 metabolism leading to homocystinemia, cystathioninemia and methylmalonic aciduria. Biochem Biophys Res Commun. 1969;35:121-6.

[8] Fischer S, Huemer M, Baumgartner M, Deodato F, Ballhausen D, Boneh A, et al. Clinical presentation and outcome in a series of 88 patients with the cblC defect. J Inherit Metab Dis. 2014;37:831-40.

[9] Weisfeld-Adams JD, Morrissey MA, Kirmse BM, Salveson BR, Wasserstein MP, McGuire PJ, et al. Newborn screening and early biochemical follow-up in combined methylmalonic aciduria and homocystinuria, cblC type, and utility of methionine as a secondary screening analyte. Mol Genet Metab. 2010;99:116-23.

[10] Cusmano-Ozog K, Lorey F, Levine S, Martin M, Nicholas E, Packman S, et al. Cobalamin $C$ disease and expanded newborn screening: The California experience. www.researchgate.net/publication/278336660_Cobalamin_c_disease_and_expanded_ne wborn_screening_The_California_experience

[11] Kim J, Gherasim C, Banerjee R. Decyanation of vitamin B12 by a trafficking chaperone. Proc Natl Acad Sci. 2008;105:14551-4.

[12] Martinelli D, Deodato F, Dionisi-Vici C. Cobalamin C defect: natural history, pathophysiology, and treatment. J Inherit Metab Dis. 2011;34:127-35.

[13] Hannibal L, Kim J, Brasch NE, Wang S, Rosenblatt DS, Banerjee R, et al. Processing of alkylcobalamins in mammalian cells: A role for the MMACHC (cblC) gene product. Mol Genet Metab. 2009;97:260-6.

[14] Kim J, Hannibal L, Gherasim C, Jacobsen DW, Banerjee R. A human vitamin B12 trafficking protein uses glutathione transferase activity for processing alkylcobalamins. $\mathrm{J}$ Biol Chem. 2009;284:33418-24.

[15] Carrillo-Carrasco N, Chandler RJ, Venditti CP. Combined methylmalonic acidemia and homocystinuria, cblC type. I. Clinical presentations, diagnosis and management. $\mathrm{J}$ Inherit Metab Dis. 2012;35:91-102.

[16] Rosenblatt DS, Aspler AL, Shevell MI, Pletcher BA, Fenton WA, Seashore MR. Clinical heterogeneity and prognosis in combined methylmalonic aciduria and homocystinuria (cblC). J Inherit Metab Dis. 1997;20:528-38.

[17] Huemer M, Scholl-Bürgi S, Hadaya K, Kern I, Beer R, Seppi K, et al. Three new cases of late-onset cblC defect and review of the literature illustrating when to consider inborn errors of metabolism beyond infancy. Orphanet J Rare Dis. 2014;9:1.

[18] Biancheri R, Cerone R, Rossi A, Schiaffino MC, Caruso U, Minniti G, et al. Early-onset cobalamin C/D deficiency: epilepsy and electroencephalographic features. Epilepsia. 2002;43:616-22.

[19] Longo D, Fariello G, Dionisi-Vici C, Cannatà V, Boenzi S, Genovese E, et al. MRI and 1H-MRS findings in early-onset cobalamin C/D defect. Neuropediatrics. 2005;36:366-72. [20] Carrillo-Carrasco N, Venditti CP. Combined methylmalonic acidemia and homocystinuria, cblC type. II. Complications, pathophysiology, and outcomes. J Inherit Metab Dis. 2012;35:103-14. 
[21] Smith SE, Kinney HC, Swoboda KJ, Levy HL. Subacute combined degeneration of the spinal cord in cblC disorder despite treatment with B12. Mol Genet Metab. 2006;88:13845.

[22] Ricci D, Pane M, Deodato F, Vasco G, Randò T, Caviglia S, et al. Assessment of visual function in children with methylmalonic aciduria and homocystinuria. Neuropediatrics. 2005;36:181-5.

[23] Gerth C, Morel CF, Feigenbaum A, Levin AV. Ocular phenotype in patients with methylmalonic aciduria and homocystinuria, cobalamin C type. Am Assoc Pediatr Ophthalmol Strabismus. 2008;12:591-6.

[24] Schimel AM, Mets MB. The natural history of retinal degeneration in association with cobalamin C (cbl C) disease. Ophthalmic Genet. 2006;27:9-14.

[25] Weisfeld-Adams JD, McCourt EA, Diaz GA, Oliver SC. Ocular disease in the cobalamin $C$ defect: A review of the literature and a suggested framework for clinical surveillance. Mol Genet Metab. 2015;114:537-46.

[26] Thauvin-Robinet C, Roze E, Couvreur G, Horellou MH, Sedel F, Grabli D, et al. The adolescent and adult form of cobalamin $C$ disease: clinical and molecular spectrum. J Neurol Neurosurg Psychiatry. 2008;79:725-8.

[27] Guigonis V, Frémeaux-Bacchi V, Giraudier S, Favier R, Borderie D, Massy Z, et al. Late-onset thrombocytic microangiopathy caused by cblC disease: Association with a factor H mutation. Am J Kidney Dis. 2005;45:588-95.

[28] Van Hove JLK, Damme-Lombaerts RV, Grünewald S, Peters H, Damme BV, Fryns $\mathrm{JP}$, et al. Cobalamin disorder Cbl-C presenting with late-onset thrombotic microangiopathy: Late-onset cobalamin C disorder. Am J Med Genet. 2002;111:195-201.

[29] Brunelli S, Meyers K, Guttenberg M, Kaplan P, Kaplan B. Cobalamin C deficiency complicated by an atypical glomerulopathy. Pediatr Nephrol. 2002;17:800-3.

[30] Geraghty MT, Perlman EJ, Martin LS, Hayflick SJ, Casella JF, Rosenblatt DS, et al. Cobalamin C defect associated with hemolytic-uremic syndrome. J Pediatr. 1992;120:9347.

[31] Brandstetter $\mathrm{Y}$, Weinhouse E, Splaingard ML, Tang TT. Cor pulmonale as a complication of methylmalonic acidemia and homocystinuria (Cb1-C type). Am J Med Genet. 1990;36:167-71.

[32] lodice FG, Di Chiara L, Boenzi S, Aiello C, Monti L, Cogo P, et al. Cobalamin C defect presenting with isolated pulmonary hypertension. Pediatrics. 2013;132:e248-51.

[33] Gündüz M, Ekici F, Özaydın E, Ceylaner S, Perez B. Reversible pulmonary arterial hypertension in cobalamin-dependent cobalamin $\mathrm{C}$ disease due to a novel mutation in the MMACHC gene. Eur J Pediatr. 2014;173:1707-10.

[34] Kömhoff M, Roofthooft MT, Westra D, Teertstra TK, Losito A, van de Kar NC, et al. Combined pulmonary hypertension and renal thrombotic microangiopathy in cobalamin $\mathrm{C}$ deficiency. Pediatrics. 2013;132:e540-e544.

[35] Profitlich LE, Kirmse B, Wasserstein MP, Diaz GA, Srivastava S. High prevalence of structural heart disease in children with cblC-type methylmalonic aciduria and homocystinuria. Mol Genet Metab. 2009;98:344-8.

[36] Lerner-Ellis JP, Tirone JC, Pawelek PD, Doré C, Atkinson JL, Watkins D, et al. Identification of the gene responsible for methylmalonic aciduria and homocystinuria, $\mathrm{cblC}$ type. Nat Genet. 2006;38:93-100.

[37] Beck BB, van Spronsen F, Diepstra A, Berger RMF, Kömhoff M. Renal thrombotic microangiopathy in patients with cblC defect: review of an under-recognized entity. Pediatr Nephrol. 2017;32:733-41.

[38] Lerner-Ellis JP, Anastasio N, Liu J, Coelho D, Suormala T, Stucki M, et al. Spectrum of mutations in MMACHC, allelic expression, and evidence for genotype-phenotype correlations. Hum Mutat. 2009;30:1072-81. 
[39] Morel CF, Lerner-Ellis JP, Rosenblatt DS. Combined methylmalonic aciduria and homocystinuria (cblC): Phenotype-genotype correlations and ethnic-specific observations. Mol Genet Metab. 2006;88:315-21.

[40] Froese DS, Krojer T, Wu X, Shrestha R, Kiyani W, von Delft F, et al. Structure of MMACHC reveals an arginine-rich pocket and a domain-swapped dimer for its $B_{12}$ processing function. Biochemistry. 2012;51:5083-90.

[41] Froese DS, Healy S, McDonald M, Kochan G, Oppermann U, Niesen FH, et al. Thermolability of mutant MMACHC protein in the vitamin B12-responsive cblC disorder. Mol Genet Metab. 2010;100:29-36.

[42] Carrillo-Carrasco N, Sloan J, Valle D, Hamosh A, Venditti CP. Hydroxocobalamin dose escalation improves metabolic control in cblC. J Inherit Metab Dis. 2009;32:728-31.

[43] Scott JM, Dinn JJ, Wilson P, Weir DG. Pathogenesis of subacute combined degeneration: a result of methyl group deficiency. Lancet Lond Engl. 1981;2:334-7.

[44] Moake JL. Thrombotic microangiopathies. N Engl J Med. 2002;347:589-600.

[45] Fakhouri F, Zuber J, Frémeaux-Bacchi V, Loirat C. Haemolytic uraemic syndrome. Lancet 2017.

[46] Serna IVA, Boedeker EC. Pathogenesis and treatment of Shiga toxin-producing Escherichia coli infections. Curr Opin Gastroenterol. 2008;24:38-47.

[47] George JN, Nester CM. Syndromes of thrombotic microangiopathy. N Engl J Med. 2014;371:654-66.

[48] Rosove MH. Thrombotic microangiopathies. Semin Arthritis Rheum. 2014;43:797-805.

[49] Baumgartner ER, Wick H, Maurer R, Egli N, Steinmann B. Congenital defect in intracellular cobalamin metabolism resulting in homocysteinuria and methylmalonic aciduria. I. Case report and histopathology. Helv Paediatr Acta. 1979;34:465-82.

[50] Koenig JC, Rutsch F, Bockmeyer C, Baumgartner M, Beck BB, Kranz B, et al. Nephrotic syndrome and thrombotic microangiopathy caused by cobalamin $\mathrm{C}$ deficiency. Pediatr Nephrol. 2015;30:1203-6.

[51] Li QL, Song WQ, Peng XX, Liu XR, He LJ, Fu LB. Clinical characteristics of hemolytic uremic syndrome secondary to cobalamin $\mathrm{C}$ disorder in Chinese children. World $\mathrm{J}$ Pediatr. 2015;11:276-80.

[52] Sharma AP, Greenberg CR, Prasad AN, Prasad C. Hemolytic uremic syndrome (HUS) secondary to cobalamin C (cblC) disorder. Pediatr Nephrol. 2007;22:2097-103.

[53] Russo P, Doyon J, Sonsino E, Ogier H, Saudubray JM. A congenital anomaly of vitamin B12 metabolism: a study of three cases. Hum Pathol. 1992;23:504-12.

[54] Chenel C, Wood C, Gourrier E, Zittoun J, Casadevall I, Ogier H. Neonatal hemolyticuremic syndrome, methylmalonic aciduria and homocystinuria caused by intracellular vitamin B12 deficiency. Value of etiological diagnosis. Arch Fr Pediatr. 1993;50:749-54.

[55] Davin JC, Buter N, Groothoff J, van Wijk J, Bouts A, Strain L, et al. Prophylactic plasma exchange in CD46-associated atypical haemolytic uremic syndrome. Pediatr Nephrol. 2009;24:1757-60.

[56] Weiss N. Mechanisms of increased vascular oxidant stress in hyperhomocysteinemia and its impact on endothelial function. Curr Drug Metab. 2005;6:27-36.

[57] Stamler JS, Osborne JA, Jaraki O, Rabbani LE, Mullins M, Singel D, et al. Adverse vascular effects of homocysteine are modulated by endothelium-derived relaxing factor and related oxides of nitrogen. J Clin Invest. 1993;91:308.

[58] Upchurch GR, Welch GN, Fabian AJ, Freedman JE, Johnson JL, Keaney JF, et al. Homocyst(e)ine decreases bioavailable nitric oxide by a mechanism involving glutathione peroxidase. J Biol Chem. 1997;272:17012-7.

[59] Rodgers GM, Conn MT. Homocysteine, an atherogenic stimulus, reduces protein C activation by arterial and venous endothelial cells. Blood. 1990;75:895-901. 
[60] Lentz SR, Sadler JE. Inhibition of thrombomodulin surface expression and protein C activation by the thrombogenic agent homocysteine. J Clin Invest. 1991;88:1906.

[61] Hajjar KA. Homocysteine-induced modulation of tissue plasminogen activator binding to its endothelial cell membrane receptor. J Clin Invest. 1993;91:2873.

[62] Hajjar KA, Mauri L, Jacovina AT, Zhong F, Mirza UA, Padovan JC, et al. Tissue plasminogen activator binding to the annexin II tail domain. Direct modulation by homocysteine. J Biol Chem. 1998;273:9987-93.

[63] Rodgers GM, Kane WH. Activation of endogenous factor $\mathrm{V}$ by a homocysteineinduced vascular endothelial cell activator. J Clin Invest. 1986;77:1909.

[64] Fryer RH, Wilson BD, Gubler DB, Fitzgerald LA, Rodgers GM. Homocysteine, a risk factor for premature vascular disease and thrombosis, induces tissue factor activity in endothelial cells. Arterioscler Thromb J Vasc Biol. 1993;13:1327-33.

[65] Wall RT, Harlan JM, Harker LA, Striker GE. Homocysteine-induced endothelial cell injury in vitro: a model for the study of vascular injury. Thromb Res. 1980;18:113-21.

[66] Starkebaum G, Harlan JM. Endothelial cell injury due to copper-catalyzed hydrogen peroxide generation from homocysteine. J Clin Invest. 1986;77:1370-6.

[67] Harker LA, Slichter SJ, Scott CR, Ross R. Homocystinemia. Vascular injury and arterial thrombosis. N Engl J Med. 1974;291:537-43.

[68] Papatheodorou L, Weiss N. Vascular oxidant stress and inflammation in hyperhomocysteinemia. Antioxid Redox Signal. 2007;9:1941-58.

[69] McCully KS. Chemical pathology of homocysteine. I. Atherogenesis. Ann Clin Lab Sci. 1993;23:477-93.

[70] McCully KS, Carvalho AC. Homocysteine thiolactone, N-homocysteine thiolactonyl retinamide, and platelet aggregation. Res Commun Chem Pathol Pharmacol. 1987;56:349-60.

[71] Mudd SH, Skovby F, Levy HL, Pettigrew KD, Wilcken B, Pyeritz RE, et al. The natural history of homocystinuria due to cystathionine $\beta$-synthase deficiency. Am J Hum Genet. 1985;37:1.

[72] Labrune P, Zittoun J, Duvaltier I, Trioche P, Marquet J, Niaudet P, et al. Haemolytic uraemic syndrome and pulmonary hypertension in a patient with methionine synthase deficiency. Eur J Pediatr. 1999;158:734-9.

[73] Bouts AH, Roofthooft MTR, Salomons GS, Davin JC. CD46-associated atypical hemolytic uremic syndrome with uncommon course caused by cblC deficiency. Pediatr Nephrol. 2010;25:2547-8.

[74] Greitz D. The hydrodynamic hypothesis versus the bulk flow hypothesis. Neurosurg Rev 2004;27:299-300.

[75] Lemoine $M$, François $A$, Grangé S, Rabant $M$, Châtelet V, Cassiman D, et al. Cobalamin $\mathrm{C}$ deficiency induces a typical histopathological pattern of renal arteriolar and glomerular thrombotic microangiopathy. Kidney Int Rep. 2018;3:1153-62.

[76] Paul EA, Guttenberg M, Kaplan P, Watkins D, Rosenblatt DS, Treat JR, et al. Atypical glomerulopathy associated with the cblE inborn error of vitamin B12 metabolism. Pediatr Nephrol. 2013;28:1135-9.

[77] Vaisbich MH, Braga A, Gabrielle M, Bueno C, Piazzon F, Kok F. Thrombotic microangiopathy caused by methionine synthase deficiency: diagnosis and treatment pitfalls. Pediatr Nephrol 2017.

[78] Noel N, Maigne G, Tertian G, Anguel N, Monnet X, Michot JM, et al. Hemolysis and schistocytosis in the emergency department: consider pseudothrombotic microangiopathy related to vitamin B12 deficiency. QJM. 2013;106:1017-22.

[79] Andrès E, Affenberger S, Zimmer J, Vinzio S, Grosu D, Pistol G, et al. Current hematological findings in cobalamin deficiency. A study of 201 consecutive patients with documented cobalamin deficiency. Clin Lab Haematol. 2006;28:50-6. 
[80] Andrès E, Affenberger S, Federici L, Korganow AS. Pseudo-thrombotic microangiopathy related to cobalamin deficiency. Am J Med. 2006;119:e3.

[81] Morath MA, Hörster F, Sauer SW. Renal dysfunction in methylmalonic acidurias: review for the pediatric nephrologist. Pediatr Nephrol. 2013;28:227-35.

[82] Baumgarter ER, Viardot C. Long-term follow-up of 77 patients with isolated methylamalonic acidaemia. J Inherit Metab Dis. 1995;18:138-42.

[83] Molteni $\mathrm{KH}$, Oberley TD, Wolff JA, Friedman AL. Progressive renal insufficiency in methylmalonic acidemia. Pediatr Nephrol. 1991;5:323-6.

[84] Walter JH, Michalski A, Wilson WM, Leonard JV, Barratt TM, Dillon MJ. Chronic renal failure in methylmalonic acidaemia. Eur J Pediatr. 1989;148:344-8.

[85] D'angio CT, Dillon MJ, Leonard JV. Renal tubular dysfunction in methylmalonic acidaemia. Eur J Pediatr. 1991;150:259-63.

[86] Cosson MA, Benoist JF, Touati G, Déchaux M, Royer N, Grandin L, et al. Long-term outcome in methylmalonic aciduria: A series of 30 French patients. Mol Genet Metab. 2009;97:172-8.

[87] Hörster F, Baumgartner MR, Viardot C, Suormala T, Burgard P, Fowler B, et al. Longterm outcome in methylmalonic acidurias is influenced by the underlying defect (mut0, mut-, cblA, cblB). Pediatr Res. 2007;62:225-30.

[88] Rutledge SL, Geraghty M, Mroczek E, Rosenblatt D, Kohout E. Tubulointerstitial nephritis in methylmalonic acidemia. Pediatr Nephrol. 1993;7:81-2.

[89] Wolff JA, Strom C, Griswold W, Sweetman F, Kulovich S, Prodanos C, et al. Proximal renal tubular acidosis in methylmalonic acidemia. J Neurogenet. 1985;2:31-9.

[90] Sauer SW, Opp S, Haarmann A, Okun JG, Kölker S, Morath MA. Long-term exposure of human proximal tubule cells to hydroxycobalamin [c-lactam] as a possible model to study renal disease in methylmalonic acidurias. J Inherit Metab Dis. 2009;32:720-7.

[91] Wahlstedt-Fröberg V, Pettersson T, Aminoff M, Dugué B, Gräsbeck R. Proteinuria in cubilin-deficient patients with selective vitamin B12 malabsorption. Pediatr Nephrol. 2003;18:417-21.

[92] Cornec-Le Gall E, Delmas Y, De Parscau L, Doucet L, Ogier H, Benoist JF, et al. Adult-onset eculizumab-resistant hemolytic uremic syndrome associated with cobalamin C deficiency. Am J Kidney Dis. 2014;63:119-23.

[93] Grangé S, Bekri S, Artaud-Macari E, Francois A, Girault C, Poitou AL, et al. Adultonset renal thrombotic microangiopathy and pulmonary arterial hypertension in cobalamin C deficiency. Lancet Lond Engl. 2015;386:1011-2.

[94] Medhioub Kaaniche F, Chaari A, Bacouch N, Bahloul M, Bouaziz M. Syndrome hémolytique et urémique de l'adulte jeune par trouble du métabolisme de la cobalamine: à propos d'un cas. Press Med. 2016;45:148-50.

[95] Carmel R, Bedros AA, Mace JW, Goodman SI. Congenital methylmalonic aciduria-homocystinuria with megaloblastic anemia: observations on response to hydroxocobalamin and on the effect of homocysteine and methionine on the deoxyuridine suppression test. Blood. 1980;55:570-9.

[96] Kind T, Levy J, Lee M, Kaicker S, Nicholson JF, Kane SA. Cobalamin C disease presenting as hemolytic-uremic syndrome in the neonatal period. J Pediatr Hematol Oncol. 2002;24:327-9.

[97] Richard E, Jorge-Finnigan A, Garcia-Villoria J, Merinero B, Desviat LR, Gort L, et al. Genetic and cellular studies of oxidative stress in methylmalonic aciduria (MMA) cobalamin deficiency type $\mathrm{C}(c b / C)$ with homocystinuria (MMACHC). Hum Mutat. 2009;30:1558-66.

[98] Menni F, Testa S, Guez S, Chiarelli G, Alberti L, Esposito S. Neonatal atypical hemolytic uremic syndrome due to methylmalonic aciduria and homocystinuria. Pediatr Nephrol. 2012;27:1401-5. 
[99] Jiménez Varo I, Bueno Delgado M, Dios Fuentes E, Delgado Pecellin C, González Meneses A, Soto Moreno A, et al. Combined methylmalonic acidemia and homocystinuria; a case report. Nutr Hosp. 2015;31:1885-8.

[100] Adrovic A, Canpolat N, Caliskan S, Sever L, Kıykım E, Agbas A, et al. Cobalamin C defect-hemolytic uremic syndrome caused by new mutation in MMACHC: Rare cause of aHUS. Pediatr Int. 2016;58:763-5.

\section{Tableau 1}

Anomalies innées du métabolisme de la cobalamine classées par phénotype biochimique

\begin{tabular}{|c|c|c|}
\hline Phénotype biochimique & $\begin{array}{l}\text { Groupe de } \\
\text { complémentation }\end{array}$ & gène \\
\hline \multirow{3}{*}{$\begin{array}{l}\text { Acidémie méthylmalonique } \\
\text { (déficit en AdoCbl) }\end{array}$} & cblA & $M M A A$ \\
\hline & cblB & $M M A B$ \\
\hline & cblD (variant 2) & $M M A D H C$ \\
\hline \multirow{4}{*}{$\begin{array}{l}\text { Acidémie méthylmalonique } \\
\text { et homocystinurie } \\
\text { (déficit en AdoCbl et MeCbl) }\end{array}$} & cblC & MMACHC \\
\hline & cblD & $M M A D H C$ \\
\hline & cblF & LMBDR1 \\
\hline & cblJ & $A B C D 4$ \\
\hline \multirow{3}{*}{$\begin{array}{l}\text { Homocystinurie } \\
\text { (déficit en MeCbl) }\end{array}$} & cblD (variant 1) & MMADHC \\
\hline & cblE & MTRR \\
\hline & cblG & MTR \\
\hline
\end{tabular}

Tableau 2

Atteintes d'organes au cours du déficit en cobalamine C

Croissance

Retard de croissance intra-utérin

Retard de croissance

Microcéphalie

Atteintes neurologiques

Crises convulsives

Troubles cognitifs

Sclérose combinée de la moelle 


\begin{tabular}{|c|c|}
\hline Atteintes ophtalmologiques & $\begin{array}{l}\text { Maculopathie } \\
\text { Rétinopathie }\end{array}$ \\
\hline Atteintes hématologiques & $\begin{array}{l}\text { Cytopénies : } \\
\text { - anémie mégaloblastique } \\
\text { - thrombopénie } \\
\text { - neutropénie }\end{array}$ \\
\hline Atteintes vasculaires & $\begin{array}{l}\text { Thromboses veineuses profondes } \\
\text { Embolies pulmonaires } \\
\text { Accidents vasculaires cérébraux }\end{array}$ \\
\hline Atteintes cardiaques & $\begin{array}{l}\text { Insuffisance cardiaque congénitale } \\
\text { Cardiopathie dilatée } \\
\text { Malformations } \\
\text { Hypertension artérielle pulmonaire }\end{array}$ \\
\hline Atteintes digestives & $\begin{array}{l}\text { Stéatose hépatique } \\
\text { Gastrite } \\
\text { Entéropathie }\end{array}$ \\
\hline Atteintes cutanées & Desquamation \\
\hline
\end{tabular}

Tableau 3

Différentes causes de microangiopathies thrombotiques

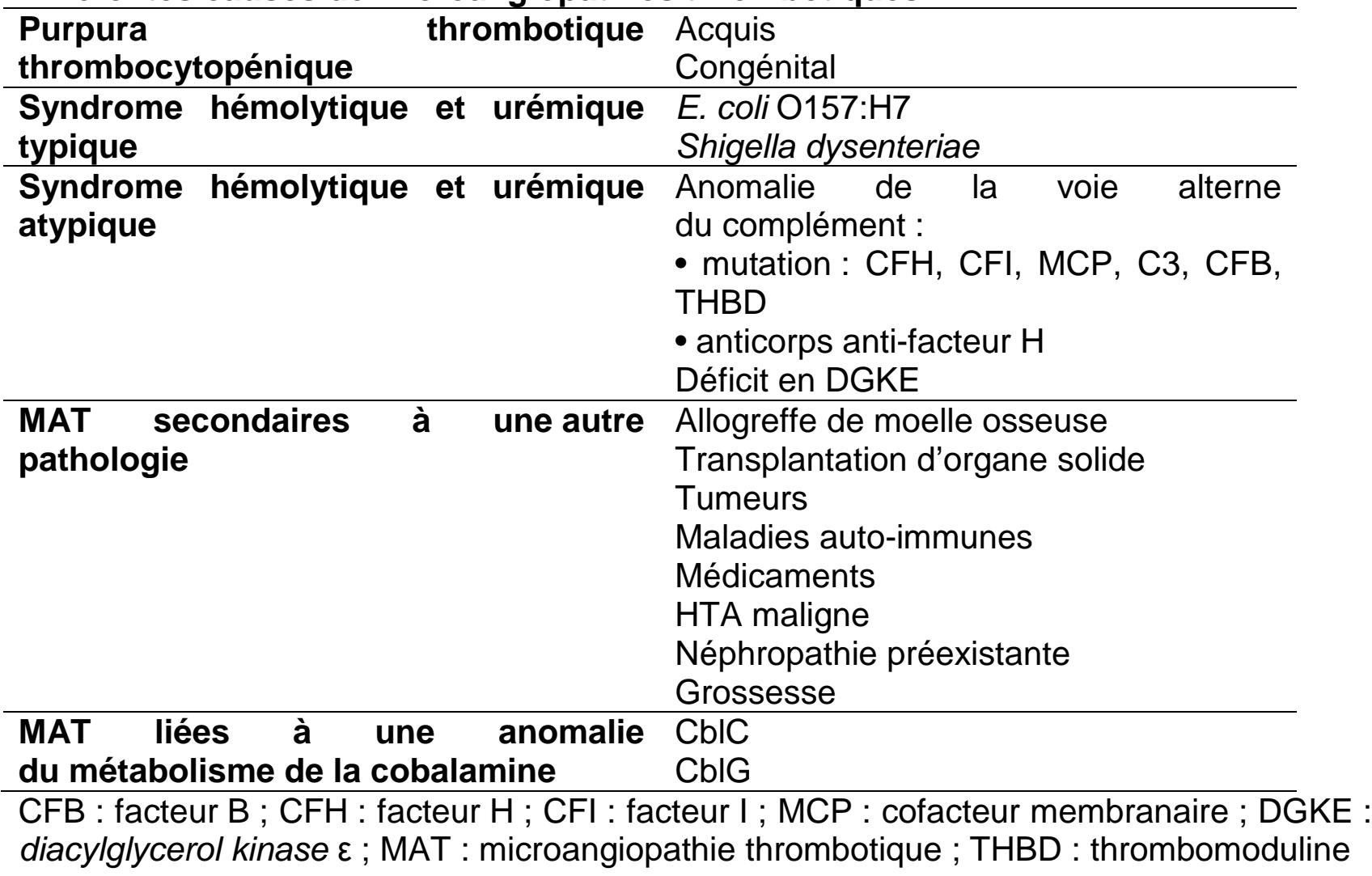




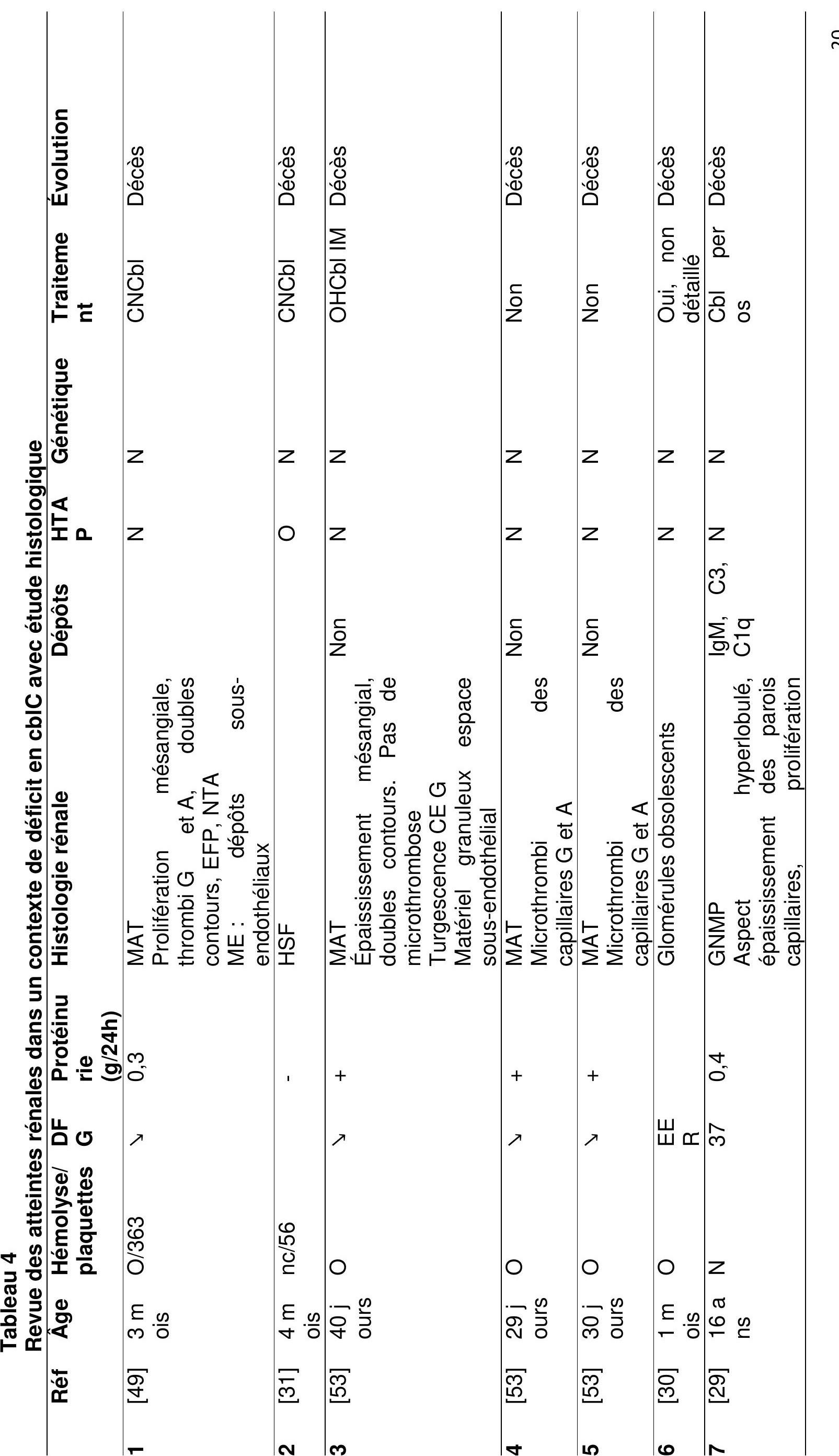




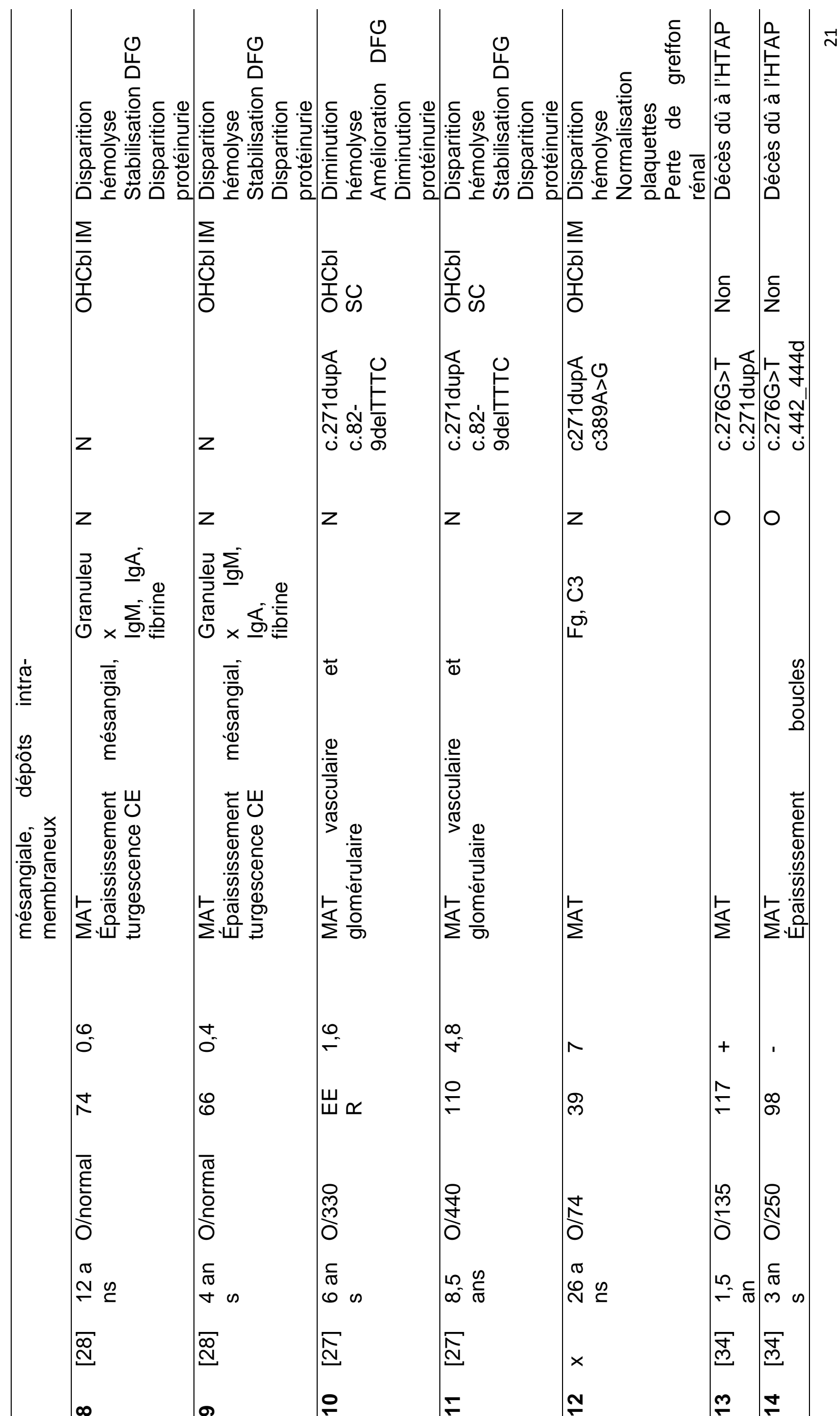




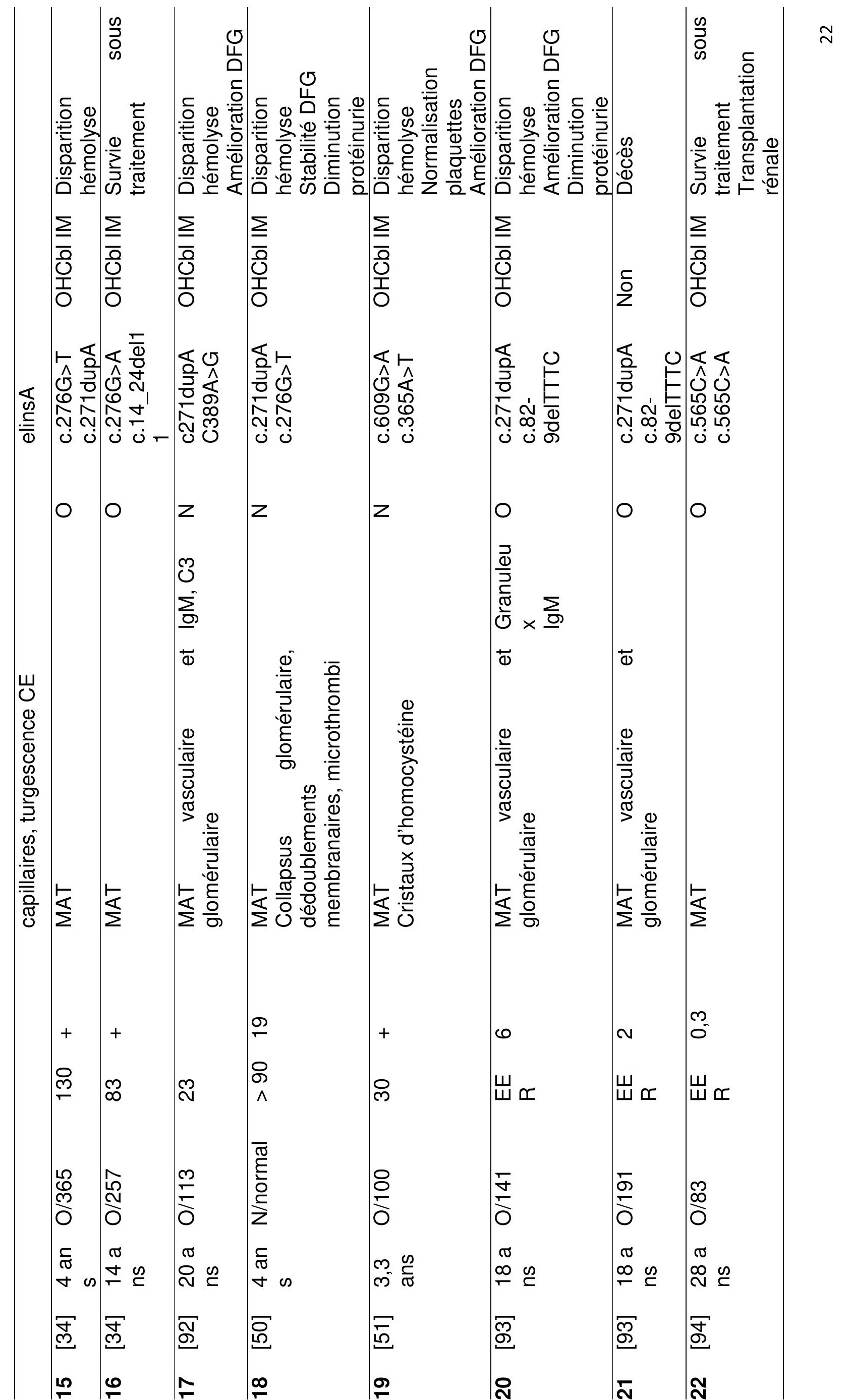




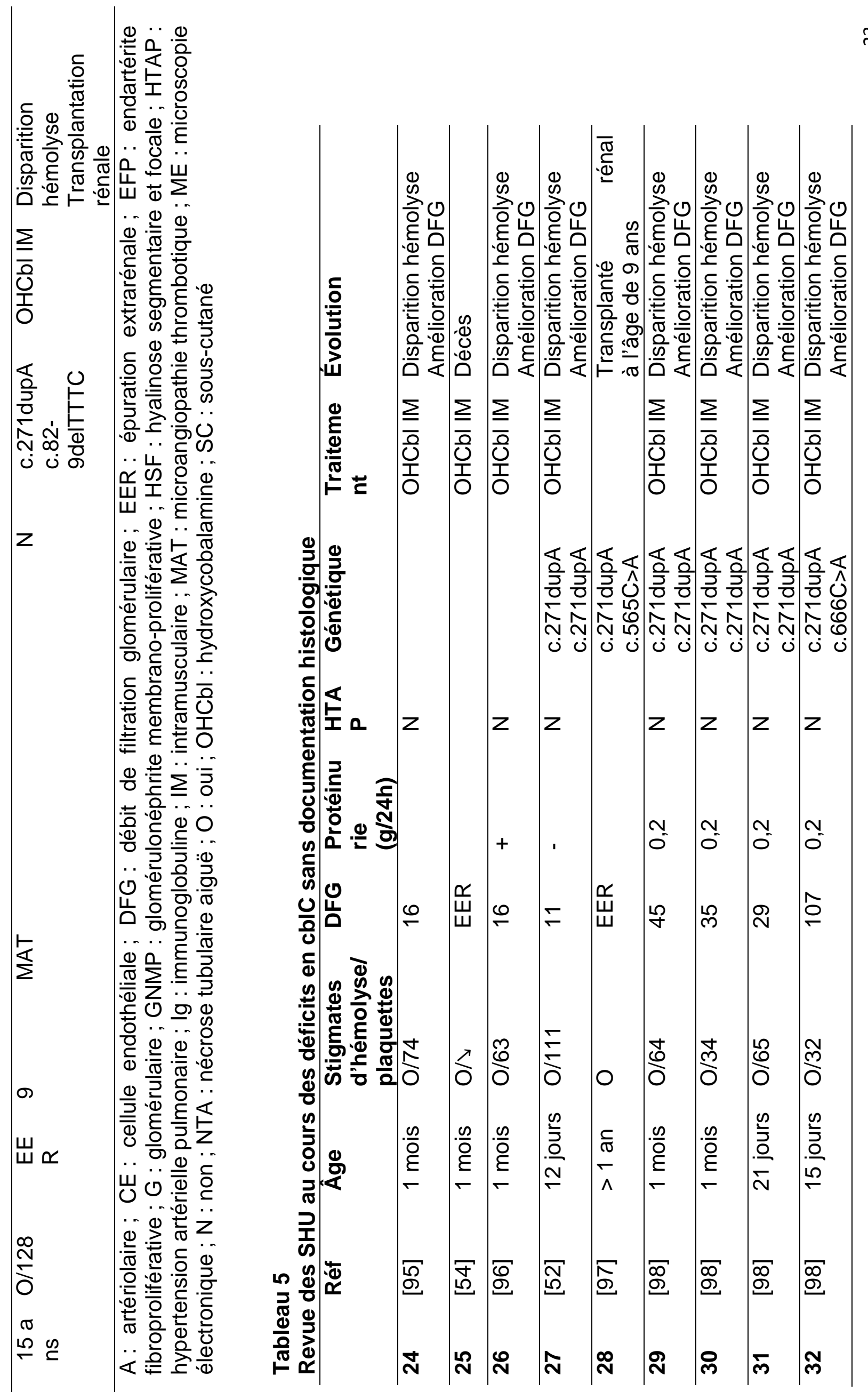




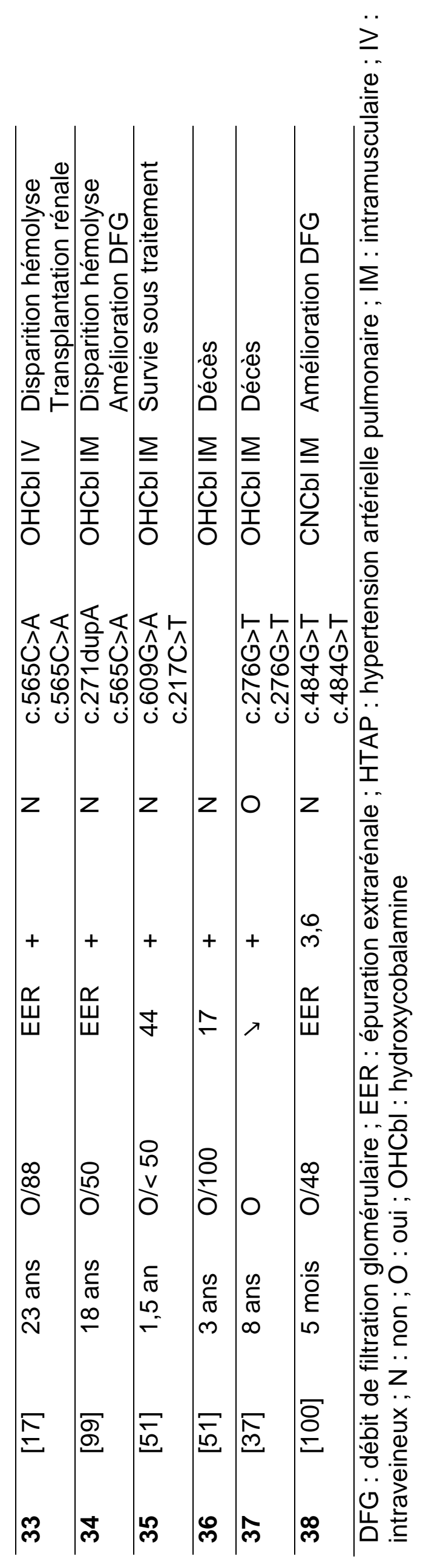


Figure 1. Structure de la cobalamine.

$\mathrm{R}=-5^{`}$-désoxyadénosyl (adénosylcobalamine), $-\mathrm{CH} 3$ (méthylcobalamine), $\mathrm{OH}$ (hydroxycobalamine) ou -CN (cyanocobalamine)

Figure 2. Métabolisme intracellulaire de la cobalamine et différents groupes de mutations.

AdoCbl: adénosylcobalamine ; Cbl: cobalamine ; CoA : coenzyme A ; MeCbl: méthylcobalamine

Figure 3. Détail du métabolisme intracellulaire de la cobalamine.

AdoCbl: adénosylcobalamine ; CoA : coenzyme A ; MeCbl : méthylcobalamine ; MMA : méthylmalonyl-coenzyme A ; MTFHR : méthylènetétrahydrofolate réductase

Figure 4. Physiopathologie du déficit en cobalamine $C$.

AdoCbl: adénosylcobalamine ; $\mathrm{CoA}$ : coenzyme A ; MeCbl : méthylcobalamine ; MMA : méthylmalonyl-coenzyme A; MTFHR ; méthylènetétrahydrofolate réductase

Figure 5. Lésions histologiques de microangiopathie thrombotique glomérulaire et artériolaire.

A-F. Biopsies rénales. Microscopie optique. A. Glomérule ischémique avec rétraction du flocculus au pôle vasculaire du glomérule (argentation de Jones; x 40). B. Microthromboses glomérulaires avec épaississement des parois capillaires glomérulaires (trichrome vert de Masson; $x$ 100). C. Mésangiolyse et congestion des boucles capillaires (trichrome vert de Masson ; x 40). D. Thrombose artériolaire (trichrome vert de Masson; x 40). E. Endartérite fibreuse avec plages fibrinoïdes d'une artère interlobulaire et épaississement des parois des capillaires glomérulaires (trichrome vert de Masson; x 20). F. Aspect de doubles contours de la membrane basale glomérulaires (argentation de Jones ; x 100). 


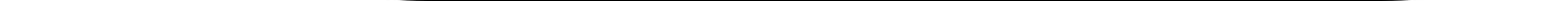




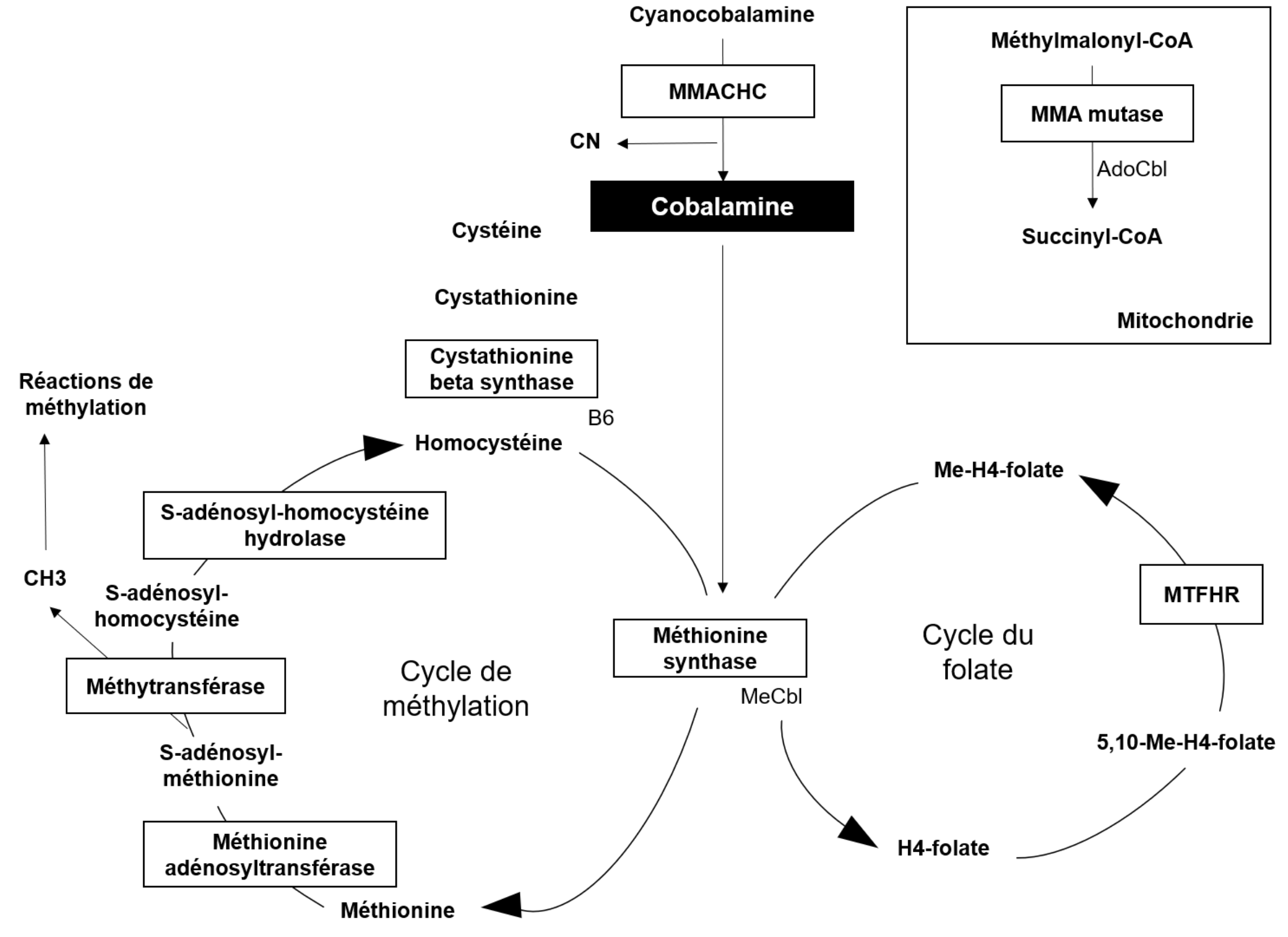




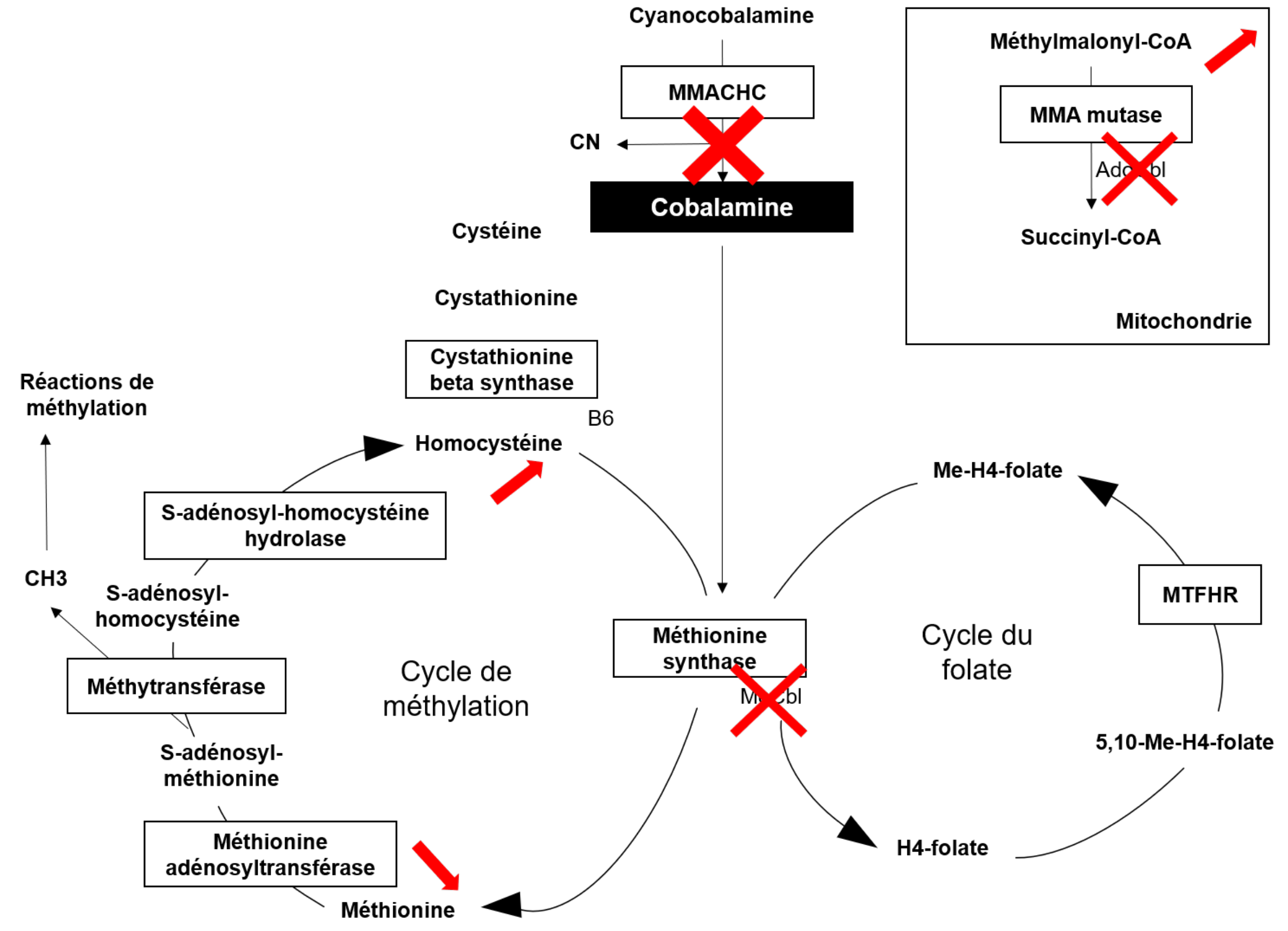



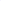